\title{
Anti-Rotational Plating for Nonisthmal Femoral Shaft Non-union after Intramedullary Nailing: A Retrospective Study
}

\author{
Xianzhi Ma', Ying Liu', Zhongshu Shan ${ }^{3}$, Qiyun Zhou ${ }^{3}$, Jianwu $\mathrm{Ma}^{3}$ and Kai Wang'* \\ ${ }^{1}$ Qinghai Provincial People's Hospital, Trauma orthopedic Department, Xining, China \\ ${ }^{2}$ Beijing Jishuitan Hospital, Medical department, Beijing, China \\ ${ }^{3}$ Qinghai Provincial People's Hospital, Trauma orthopedic Department, Xining, China
}

Submission: December 14, 2019; Published: January 10, 2020

*Corresponding author: Kai Wang, Qinghai Provincial People's Hospital, Trauma orthopedic Department, Xining, 810000, China

\begin{abstract}
Objective: This study considered the success of anti-rotational plating (ARP) with a retained nail left in situ for nonisthmal femoral shaft nonunion after femoral nailing.

Methods: Fifty-three patients presenting with nonisthmal femoral shaft nonunion after femoral nailing were selected (according to inclusion criteria) for a retrospective study from April 2012 to June 2016. All patients received treatment with ARP entailing a nail left in situ and combined with bone grafting. Main out measures include operation time, amount of bleeding during operation, union time and union rate. Follow-ups, including radiological and clinical function examinations, were carried out at 1, 2, 3, 4, 6, and 12 months after surgery and then once a year, to monitor callus growth and functional recovery of the injured limb.

Results: Mean operation time was $99.3 \pm 27.8 \mathrm{mins}$ (range $=50-215 \mathrm{mins}$ ), and mean blood loss was $494.9 \pm 281.3 \mathrm{ml}(20-1400 \mathrm{ml})$ The average follow-up time was 18.7 months (12-26 months). All patients in the current study achieved bone union (100\%). We observed no postoperative complications, such as failure of internal fixation, infections, angular or rotational malunion. Physicians removed the intramedullary nail and plate from five patients 11-16 months after the operation without any refracture. During the last follow-up, all patients could flex their knees more than $100^{\circ}$.
\end{abstract}

Conclusion: Anti-rotational plating with a nail left in situ, combined with bone grafting, is an effective treatment option for nonisthmal femoral shaft nonunions.

Keywords: Anti-Rotational Plating; Nonisthmal Femoral Shaft; Bone Grafting; Nonunion

Abbreviations: IM: Intramedullary nailing; ABG: Autologous Bone Grafting; ARP: Anti-Rotational Plate

\section{Introduction}

Intramedullary nailing (IM) is the primary method for treating nonunion of femoral shaft fractures [1]. However, as the IM technique has become more common, clinical results have suggested that the incidence of femoral shaft fracture nonunion is around 0.8-2\% [2]. Exchange intramedullary nailing is currently the gold standard treatment for femoral shaft fracture nonunion, and it sees a cure rate of $70-100 \%[1,3]$, but several reports have suggested it may increase risk of future femoral shaft fracture nonunion [4,5]. Ueng et al. [6] first reported that reversed IM and additional plate fixation, combined with autologous bone grafting (ABG), is an effective treatment considering its successful union rate of $100 \%$. This has therefore become a method that orthopedists frequently use in clinical practice. Zhang et al. [7] stated that the main mechanical function of the additional plate is to provide rotational stability for the fracture site, which led to its anti-rotational plate (ARP) designation. We agree with this analysis of ARP. The purpose of the current study was to observe the results of an ARP when the IM is left in situ as a treatment for nonisthmal femoral shaft nonunion following femoral nailing.

\section{Materials and Methods}

The current study collected clinical data of 53 patients with nonisthmal femoral shaft nonunion after femoral nailing that visited our hospital from April 2012 to June 2016. The Orthopedic Trauma Association classification helped define the whole femoral shaft length from the level of the lesser trochanter's transverse 
lower edge to the upper edge of the transepicondyla. Moreover, the nonunion site of the femoral shaft fracture occurs in one of three parts of the femur: supraisthmal, isthmal, and infraisthmal [8]. Our study includes 35 males and 18 females with an average age of 30.8 years (range: 21-54). Among these are 30 cases of traffic accident injuries, 17 cases of high falling injuries and six cases of fall damage. Using Winquist-Hansen classification, we classified the original fractures as 31 cases of Type I, 17 cases of Type II and five cases of Type III. There were 44 cases of a closed fracture, four cases of multiple fractures, one case of an ipsilateral radius fracture, one case of a contralateral tibia and fibula fracture and three cases of open fractures including two cases of Type I and one case of Type II using the Gustilo-Anderson classification. The average diameter of the IM in original repair was $10.4 \mathrm{~mm}$ (9$12 \mathrm{~mm}$ ) and mean union time was 22.2 months (10-59 months).

\section{Compliance with Ethical Standards}

The Ethics Committee of our hospital approved this study. Any related procedures were performed in accordance with relevant guidelines and regulations. Informed consent was obtained from all individual participants included in the study.

\section{Diagnosis Criteria}

One of the diagnosis criteria was persistent pain at the fracture site, potentially worsened by mobilization or weightloading following at least 9 months after IM fixation. Patients felt pressing pain at the fracture site without apparent axial abnormal movement. X-ray films showed sclerotic margins without continuous callusing across the fracture site or a failure to develop callus formation in at least three cortices at the fracture position [7]. No apparent bone absorption, osteopenia or bone defect was present at the broken ends of the fracture, nor was there any breakage or failure of the IM nail itself. There were no signs of increased callusing for 3 months.

\section{Inclusion criteria}

i. Age from 18 to 65 years;

ii. Nonisthmal femoral shaft non-union after IM;

iii. ARP with a nail left in situ.

\section{Exclusion criteria}

i. The original fracture was a pathological fracture;

ii. The original fracture was Type III B or Type III C according to the Gustilo-Anderson classification;

iii. Local soft tissue infection or infectious nonunion;

iv. Patients with a non-union gap exceeding $3 \mathrm{~cm}$ and an area more than $50 \%$ that required open grafting;

v. Pregnant women;

vi. Patients with severe systemic diseases, such as cardiovascular and cerebrovascular diseases, and urinary system diseases;

vii. Patients who recently adopted use of corticosteroids or immunosuppressors.

\section{Surgical skill}

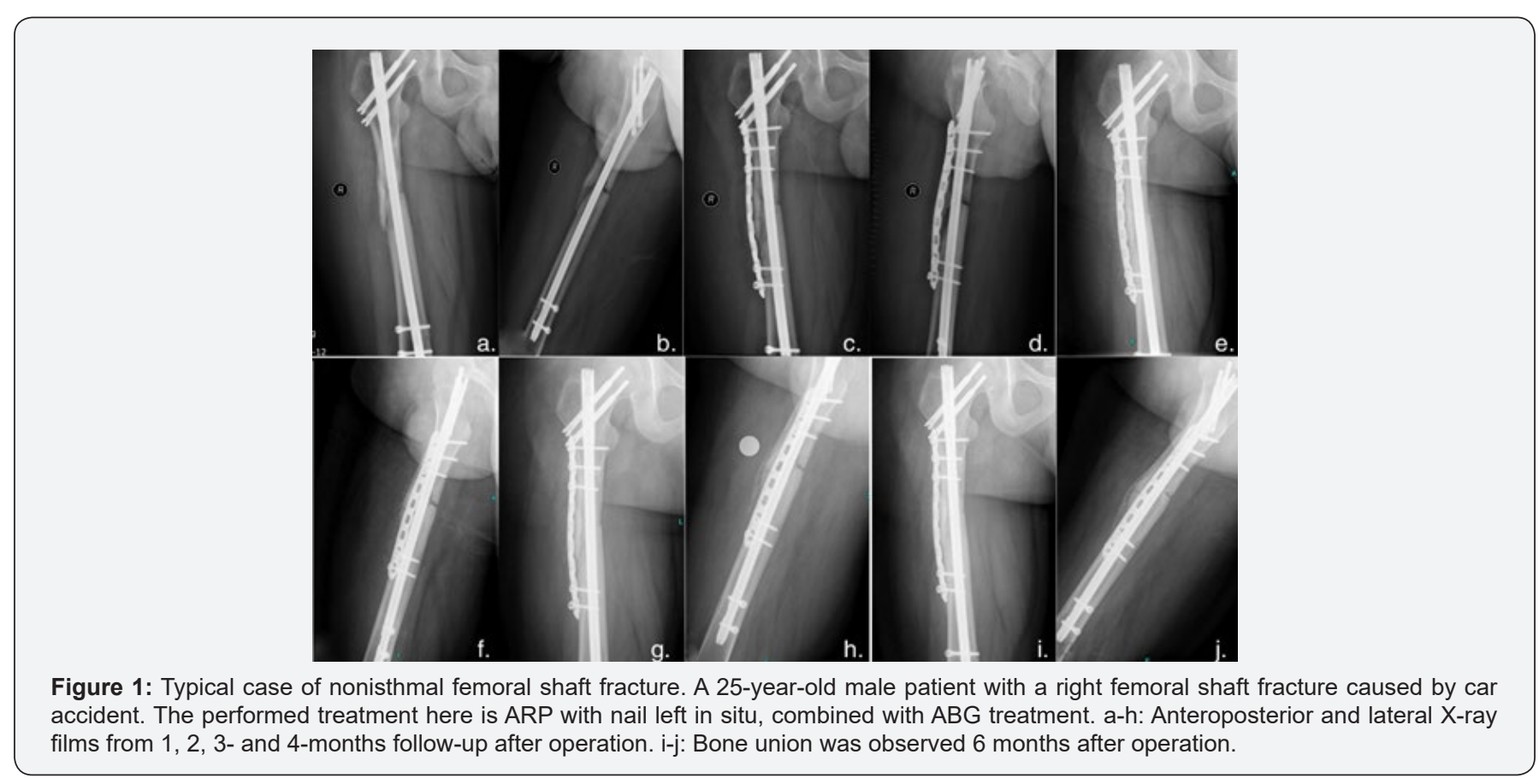


ARP internal fixation with a nail left in situ, combined with bone grafting. Adopting a lateral approach and exposing the nonunion site, the physician performed ARP with ABG. The physician rotated the distal femur under a flexed knee position to check rotational instability, the removed fibrous tissue and performed decortication around the nonunion site. A $3.5 \mathrm{~mm}$ titanium alloy locking plate (Synthes USA) was used, and two or three nonlocked screws were placed off-center to avoid the in-situ IM nail. In most patients, bicortical screw leverage was completed in the metaphysis, but sometimes unicortical leverage was performed in the diaphysis, involving placing at least three oblique screws in the diaphyseal fragment. Cancellous bone bulks were impacted into the bone gap and the strips implanted around the nonunion site. A physician collected aerobic and anaerobic cultures from the non-union site to confirm no infection was present. In the case of detected infection, the physician prescribed antibiotics according to the result of bacterial culture, who were not included in the study. One typical case of using this skill for treating nonisthmal femoral shaft fracture was presented in Figure 1.

\section{Postoperative management and follow-up}

The drainage tube was removed 2 days after the operation, at which point isometric contraction of quadriceps and other leg muscles began. Hip and knee-joint exercises began on the first day after the operation. Patients were informed they could increase their activity levels and bear partial weight after the operation based on clinical and radiographic evidence of healing to the preoperative level. Follow-up was carried out at 1, 2, 3, 4, 6, and 12 months, and afterwards at least once a year, including observing the range of movement of the knee-joint and the growth of bone callus [9]. This study adopted the lower limb function standard of the American Academy of Orthopedic Surgeons to assess patients' function scores after bone union. The diagnostic criterion of bone union included when radiology showed bridging callus formation of at least three cortices in the fracture site and painless full-weight bearing was possible. Malalignment was defined as an angular deformity exceeding 5 degrees, while malrotation deformity meant greater than 15 degrees and a length discrepancy referred to more than $2 \mathrm{~cm}$.

\section{Statistical Analysis}

The current study employed the Statistical Package for the Social Sciences (SPSS) 19.0 (SPSS, Chicago, USA) for its statistical analysis and the Fisher exact test to evaluate categorical variables. A P-value $<0.05$ was considered statistically significant.

\section{Results}

The mean operation time was $99.3 \pm 27.8 \mathrm{~min}$ (50 215 mins) and the average amount of bleeding was $494.9 \pm 281.3 \mathrm{ml}(20-1400$ $\mathrm{ml}$ ). The average follow-up time was 18.7 months (12-26 months). All patients achieved bone union by 6.4 months clinically (4-8 months) on average and 5.2 months radiologically (2-16 months), without any intervening measures during follow-up. None of the patients experienced internal fixation loosening or breakage, nor did they experience infection, or angular or rotational malunion. There was no refracture after removing the IM nail and plate 11-16 months after the operation for five patients, while others recovered to normal activity without removing the internal fixation during follow-up. During the final follow-up, all patients could flex their knees $>100^{\circ}$ (Table 1 ).

Table 1: Details of treatment.

\begin{tabular}{|c|c|}
\hline \multicolumn{2}{|c|}{ Patient gender } \\
\hline Males & 35 \\
\hline Females & 18 \\
\hline Mean (range) Age & 30.8 years $(21-54)$ \\
\hline \multicolumn{2}{|l|}{ Type of non-union } \\
\hline Hypertrophic nonunion & 44 \\
\hline Atrophic nonunion & 7 \\
\hline Delayed union & 2 \\
\hline \multicolumn{2}{|l|}{ Primary fracture type } \\
\hline Closed & 44 \\
\hline Open & 3 \\
\hline Combined with other fracture & 6 \\
\hline \multicolumn{2}{|l|}{$\begin{array}{l}\text { Winquist Hansen fracture } \\
\text { classification }\end{array}$} \\
\hline Type 1 & 31 \\
\hline Type 2 & 17 \\
\hline Type 3 & 5 \\
\hline \multicolumn{2}{|l|}{ Fracture site } \\
\hline Supraisthmal & 31 \\
\hline Infraisthmal & 22 \\
\hline \multicolumn{2}{|l|}{ Primary intervention } \\
\hline $\begin{array}{l}\text { Antegrade Locked } \\
\text { intramedullary nail }\end{array}$ & 41 \\
\hline $\begin{array}{l}\text { Retrograde Locked } \\
\text { intramedullary nail }\end{array}$ & 12 \\
\hline \multicolumn{2}{|l|}{ Dynamization } \\
\hline Prior & 15 \\
\hline At time of surgery & 11 \\
\hline
\end{tabular}




\section{Discussion}

Exchange IM has been the gold standard of treatment for femoral shaft fracture nonunion, considering it regularly sees a cure rate of $72-100 \%[2,3]$. However, Banaszkiewicz \& Weresh et al. [5] found failure rates as high as $73 \%$ in the wake of using this method. Park et al. $[8,10]$ also confirmed the method has a higher failure rate for nonisthmal femoral nonunion, which is typically caused by mechanical instability. In his study, Ueng [6] discovered that IM could not provide enough rotational stability in nonisthmal femoral nonunion. To tackle this issue, Ueng [6] proposed retaining the original IM and adding plates, along with ABG treatment. In his study, 17 patients subsequently achieved bone union with $100 \%$ union rate. Following him, Park et al. [11] likewise reported similar clinical efficacy in the process of using this method. Zhang et al. [7] stated that the plate succeeds by enhancing mechanical stability, while the main cause of femoral shaft fracture nonunion is mechanical instability. Retaining the original IM also guarantees axial stability and bending stability. Moreover, ARP improves the rotational stability at the fracture ends and provided the crucial mechanical elements for local fiber cartilage calcification and promotion of callus preliminary connection. We agree with Zhang's view for the following reasons. On the one hand, in the current study, nonunion mainly occurred at nonisthmal femoral shaft locations. From an anatomical perspective, medullary space was not at issue in nonunions because even the largest intramedullary nail can only provide axial stability and partial rotational stability. The current study therefore proposes using the additional plate to cope with rotational instability, rather than providing axial stability. Most nonunion cases in our study were hypertrophic nonunion caused primarily by poor local mechanical stability resulting from IM nails failing to provide enough rotational stability. The ARP could provide such rotation-resilient stability, which is a vital mechanical factor for calcifying local fibrous cartilage and preliminarily connecting callusing [11]. According to Park et al. [12], ARP with a nail left in situ has 3.3 times the rotation resistance and 2.6 times the bending strength when compared with EN. Finally, the biological mechanism of this method, using ABG at fracture ends to promote bone remodeling, stimulated local osteogenesis, bone conduction and bone induction.

When one compares ARP to EN, the ARP treatment has many advantages, including minimal invasion, short operation time, a high bone healing rate, and generally satisfactory outcomes. Moreover, the biological mechanism in augmentation plating surgery stimulates bone remodeling at the fracture ends through successful bone autografts with osteogenesis, osteoconduction, and osteoinduction. Several studies [13-16] have shown that augmentation plating surgery has a plenitude of advantages over EN for treating long bone nonunions after failed IM; ARP with $\mathrm{ABG}$ also showed excellent union rate in this current study.
Considering the small size of the plates, ARP may add some stability to the extant IM nail. We believe that ABG is essential in the bone union for this technique. Theoretically, for hypertrophic femoral shaft nonunions, ABG is not an essential step. However, decortication and bone grafting must occur in all cases $[17,18]$ because hypertrophic calluses tend to become damaged during operation. The result is that most patients prefer a more proactive treatment option. Moreover, ABG can stimulate the reoccurrence of the osteogenesis mechanism, increase the possibility of bone union and decrease reoperation rates. In the present study, all nonunions treated with ARP and ABG achieved bone union. As a result, we suggest $A B G$ and strong mechanical fixation play a key role in this success and thus we believe that ARP with ABG is a better option than EN for nonisthmal femoral nonunions, while one can easily use ARP to treat femur malrotation.

Some related literature has reported that EN and ARP achieve a good curative effect. Yang et al. [19] retrospectively analyzed 41 cases of femoral shaft nonunions that were treated with EN and reported that the cure rate of isthmus nonunion was $87 \%$, while nonisthmus non-union achieved $50 \%$ bone union. Brinker and $\mathrm{O}^{\prime}$ Connor [3] found the absolute indication for EN was the nonunion of femoral shaft (noncomminuted or noninfectious), while EN could not play an effective role in bone grafting or improving the stability of femoral metaphysis fractures and nonunion of multiple isthmic fractures.

The current study has some limitations. For example, as a retrospective study, patient selection bias is a possibility; a prospective, large-scale, randomized trial examining the anatomic levels is a way to mitigate this limitation and move forward with this topic in future studies. Moreover, although the current study was balanced by selecting as many cases as possible over as long a time frame as possible, there were no parallel control studies on large sample sizes at home and abroad owing to low incidence rate. There were also differences in terms of femoral fracture site, fracture type, bone defect size of nonunion, surgeons' experience in bone grafting, etc. Unified inclusion and exclusion criteria and standardized quantitative indicators were therefore something this study could not establish.

\section{Conclusion}

In conclusion, affixing an ARP and leaving the IM nail in situ, combined with bone grafting in treating nonisthmal femoral shaft nonunions, is a recommended treatment espousing the advantages of a simple operation technique, short operation time, less bleeding, light trauma, and a satisfactory clinical curative effect.

\section{Acknowledgement}

We thank Ms. Junya Zhao for her assistance in language editing. 


\section{Conflict of Interest}

All authors declare that they have no conflict of interest.

\section{References}

1. Sean E Nork. Fractures of the shaft of the femur. In: Bucholz, Robert W, etc, editors. ( $6^{\text {th }}$ Edn), Rockwood \& Green's fracture in Adults.

2. Winquist RA, Hansen ST Jr, Clawson DK (1984) Closed intramedullary nailing of femoral fractures. A report of five hundred and twenty cases. J Bone Joint Surg Am 66(4): 529-539.

3. Brinker MR, O'Connor DP (2007) Current Concepts Review: Exchange Nailing of Ununited Fractures. J Bone Joint Surg Am 89(1): 177-188.

4. Banaszkiewicz PA, Sabboubeh A, McLeod I, Maffulli N (2003) Femoral exchange nailing for aseptic non-union: not the end to all problems. Injury 34(5): 349-356.

5. Weresh MJ, Hakanson R, Stover MD, Sims SH, Kellam JF, et al. (2000) Failure of exchange reamed intramedullary nails for ununited femoral shaft fractures. J Orthop Trauma 14: 335-338.

6. Ueng SWN, Chao EK, Lee SS, Shih CH (1997) Augmentative plate fixation for the management of femoral nonunion after intramedullary nailing. J Trauma 43(4): 640-644.

7. Gang, zhao, Bosong, zhang, Lin, et al. (2009) The diagnosis and treatment of femoral shaft fracture nonunion was caused by the rotational instability after the treatment of locked intramedullary nail. Chinese journal of surgery 16: 1232-1235.

8. Park J, Kim SG, Yoon HK, Yang KH (2010) The treatment of nonisthmal femoral shaft nonunions with im nail exchange versus augmentation plating. J Orthop Trauma 24: 89-94.

9. Ohanson NA, Liang MH, Dahroy L (2004) Assessment instruments, Reliability, validity, and sensitivity to change America Academy of Orthopaedic Surgeons lower limb outcomes. J Bone Joint Surg Am 86(5): 902-909.
10. Yang KH, Kim JR, Park J (2012) Nonisthmal femoral shaft nonunion as a risk factor for exchange nailing failure. J Trauma Acute Care Surg 72(2): E60-64.

11. Park J, Kim SG, Yoon HK, Yang KH (2010) The treatment of nonisthmal femoral shaft nonunions with im nail exchange versus augmentation plating. J Orthop Trauma 24(2): 89-94.

12. Park K, Kim K, Choi YS (2011) Comparison of mechanical rigidity between plate augmentation leaving the nail in situ and interlocking nail using cadaveric fracture model of the femur. Int Orthop 35(4): 581-585.

13. Chiang TC, Johnson JE, Tarkin IS, Siska PA, Farrell DJ, Mormino MA (2016) Plate augmentation for femoral nonunion : more than just a salvage tool ? Arch Orthop Trauma Surg 136(2): 149-156.

14. Birjandinejad A, Ebrahimzadeh MH, Ahmadzadeh Chabock H (2009) Augmentation plate fixation for the treatment of femoral and tibial nonunion after intramedullary nailing. Orthopedics 32(6): 409.

15. Park J, Yang KH (2013) Indications and outcomes of augmentation plating with decortication and autogenous bone grafting for femoral shaft nonunions. Injury 44(12): 1820-1825.

16. Said GZ, Said HG, El-Sharkawi MM (2011) Failed intramedullary nailing of femur : open reduction and plate augmentation with the nail in situ. Int Orthop 35(7): 1089-1092.

17. Chen CM, Su YP, Hung SH, Lin CL, Chiu FY (2010) Dynamic compression plate and cancellous bone graft for aseptic nonunion after intramedullary nailing offemoral fracture. Orthopedics 33(6): 393.

18. Hakeos WM, Richards JE, Obremskey WT (2011) Plate fixation of femoral nonunions over an intramedullary nail with autogenous bone grafting. J Orthop Trauma 25(2): 84-89.

19. Yang KH, Kim JR, Park J (2012) Nonisthmal femoral shaft nonunion as a risk factor for exchange nailing failure. J Trauma Acute Care Surg 72(2): E60-64.

\section{Your next submission with Juniper Publishers} will reach you the below assets

- Quality Editorial service

- Swift Peer Review

- Reprints availability

- E-prints Service

- Manuscript Podcast for convenient understanding

- Global attainment for your research

- Manuscript accessibility in different formats

( Pdf, E-pub, Full Text, Audio)

- Unceasing customer service

Track the below URL for one-step submission https://juniperpublishers.com/online-submission.php 